\title{
IS THERE ROOM FOR IMPROVING CASE MANAGEMENT FOR CONTACTS OF MENINGOCOCCAL DISEASE IN THE EUROPEAN UNION?
}

P L Lopalco (pierluigi.lopalco@ecdc.europa.eu) ${ }^{1}$

1.Programme on Vaccine-Preventable Diseases, European Centre for Disease Prevention and Control, Stockholm, Sweden

Invasive meningococcal disease (IMD) is a severe illness primarily affecting children and young adults. It has a high case fatality rate (10\%-14\%) and $11 \%-19 \%$ of patients who recover experience permanent hearing loss, mental retardation, loss of limbs or other serious sequelae [1]. Every individual meningococcal case, therefore, is an important public health issue; furthermore, meningococcal infection is a severe threat because of the possibility of generating clusters among close contacts (school, work, household, etc.).

To date, safe and effective vaccines have been developed for serogroups A, C, W135 and Y. Immunisation programmes have also been effective in reducing the overall burden of disease. However, a vaccine against $B$ serotype is still far from coming on the market and universal vaccination strategies using the meningococcal $C$ vaccine have only been implemented in a few European countries.

For this reason, secondary preventive measures - regarding public health management of cases and their close contacts - are still paramount.

In this issue of Eurosurveillance, Hoek and colleagues [2] report results of an interesting survey that reveals several gaps in this field, including on the use of antibiotics in children and pregnant women, uncertainties in defining "close contacts" and inertia in national policy changes reaching the local level.

In another article in today's issue, Stefanoff and colleagues [3] - focusing only on household contacts - underline again the need for clear evidence-based recommendations for public health case management and surveillance. Although the preliminary data suggest that the situation has improved in 2007, the proportion of cases in which chemoprophylaxis was administered to close contacts in Poland is still not satisfactory.

Finally, a recent article by Petsas and colleagues [4] on a case of IMD in a health care worker raised an interesting discussion highlighting once more the lack of a common agreement on how to define "close contacts" of IMD cases. Fusco and Puro, in a letter to the Editor [5], suggest that ambulance workers giving assistance to a suspect IMD case should be considered at high risk and be offered chemoprophylaxis, especially when they are not advised to wear face masks.

In conclusion, we believe that in this field there is a clear need for evidence-based public health policies and recommendations that should be effectively communicated and implemented on the basis of a large consensus at national and regional level.

The European Centre for Disease Prevention and Control is committed to supporting European Union Member States in developing the best policies on public health management for cases of IMD and their contacts, filling the gaps highlighted by several scientists in the field.

\section{References}

1. Centers for Disease Control and Prevention, United States. Meningococcal Disease. Fact-sheet. March 30 2006. Available from: http://www.cdc.gov/ncidod/ $\mathrm{dbmd/diseaseinfo/meningococcal \_ t.htm}$

2. Hoek M, Hanquet G, Heuberger S, Stefanoff P, Zucs P, Ramsay M, et al. A European survey on public health policies for managing cases of meningococcal disease and their contacts. Euro Surveill 2008;13(10). Available from: http://www. eurosurveillance.org/edition/v13n10/080306_4.asp

3. Stefanoff P, Rosinska M, Karczewski G, Zielinski A. The detection of meningococcal household clusters and their prophylaxis in the changing epidemiological situation of invasive meningococcal disease in Poland. Euro Surveill 2008;13(10). Available from: http://www.eurosurveillance.org/edition/ v13n10/080306 3.asp

4. Petsas A, Sharma A, Aghadiuno 0, Abid M, Paranthaman K. A secondary case of meningococcal disease in an ambulance worker, Berkshire, November 2007. Euro Surveill 2008;13(4). Available from: http://www.eurosurveillance.org/ edition/v13n04/080124_1.asp

5. Fusco FM, Puro V. Letter: Meningococcal disease in an ambulance worker. Euro Surveill 2008:13(10). Available from: http://www.eurosurveillance.org/edition/ v13n10/080306_5.asp

This article was published on 6 March 2008.

Citation style for this article: Lopalco PL. Is there room for improving case management for contacts of meningococcal disease in the European Union?. Euro Surveill. 2008;13(10):pii=8057. Available online: http://www.eurosurveillance.org/ViewArticle. aspx?ArticleId $=8057$ 